\title{
As the raven flies: using genetic data to infer the history of invasive common raven (Corvus corax) populations in the Mojave Desert
}

\author{
ROBERT C. FLEISCHER,$*$ WILLIAM I. BOARMAN ${ }^{\dagger}$ ELENA G. GONZALEZ, $* \$$ \\ ALVARO GODINEZ, ${ }^{*} \xi$ KEVIN E. OMLAND, $\dagger$ SARAH YOUNG,${ }^{* \psi}$ LAUREN \\ HELGEN,* GRACIA SYED, * and CARL E. MCINTOSH* ${ }^{*}$
}

*Center for Conservation and Evolutionary Genetics, National Zoological Park \& National Museum of Natural History, Smithsonian Institution, Washington, DC 20008, USA; ${ }^{\dagger}$ Conservation Science Research \& Consulting, 2522 Ledgeview Place, Spring Valley, CA 91977, USA; †Department of Biological Sciences, University of Maryland, Baltimore County, Baltimore, MD 21250 USA.

Correspondence: Robert C. Fleischer, CCEG, Science Building, NZP, PO BOX 37012, MRC 5503, Washington, DC 20008, USA. E-mail: fleischerr@si.edu

${ }^{\S}$ Present address: Departamento de Biodiversidad y Biología Evolutiva, Museo Nacional de Ciencias Naturales, CSIC, José Gutiérrez Abascal, 2; 28006 Madrid, Spain.

${ }^{\xi}$ Present address: University of Maryland Biotechnology Institute (UMBI), 9600 Gudelsky Drive, Rockville, Md 20850 USA.

$\psi$ Present address: Pennsylvania Association for Sustainable Agriculture, 650 Smithfield Street, Suite 210, Pittsburgh, PA 15222 USA.

+ Present address: Laboratory of Genomic Diversity, National Cancer Institute, Building 560, Room 21-23, Frederick, MD 21702-1201 USA. 


\section{Abstract}

Common raven (Corvus corax) populations in Mojave Desert regions of southern California and Nevada have increased dramatically over the past five decades. This growth has been attributed to increased human development in the region, as ravens have a commensal relationship with humans and feed extensively at landfills and on roadkilled wildlife. Ravens, as a partially subsidized predator, also represent a problem for native desert wildlife, in particular threatened desert tortoises (Gopherus agassizi). However it is unclear whether the more than 15 -fold population increase is due to in situ population growth or to immigration from adjacent regions where ravens have been historically common. Ravens were sampled for genetic analysis at several local sites within five major areas: the West Mojave Desert (California), East Mojave Desert (southern Nevada), southern coastal California, northern coastal California (Bay Area), and northern Nevada (Great Basin). Analyses of mtDNA control region sequences reveal an increased frequency of raven "Holarctic clade" haplotypes from south to north inland, with "California clade" haplotypes nearly fixed in the California populations. There was significant structuring among regions for mtDNA, with high $\mathrm{F}_{\mathrm{ST}}$ values among sampling regions, especially between the Nevada and California samples. Analyses of eight microsatellite loci reveal a mostly similar pattern of regional population structure, with considerably smaller, but mostly significant, values. The greater mtDNA divergences may be due to lower female dispersal relative to males, lower $\mathrm{N}_{\mathrm{e}}$, or effects of high mutation rates on maximal values of $\mathrm{F}_{\mathrm{ST}}$. Analyses indicate recent population growth in the West Mojave Desert and a bottleneck in the northern California populations. While we cannot rule out in situ population growth as a factor, patterns of movement inferred 
from our data suggest that the increase in raven populations in the West Mojave Desert resulted from movements from southern California and the Central Valley. Ravens in the East Mojave Desert are more similar to ones from northern Nevada, indicating movement between those regions. If this interpretation of high gene flow into the Mojave Desert is correct, then efforts to manage raven numbers by local control may not be optimally effective.

Keywords: ravens, Corvus, invasive species, control region, microsatellites, Mojave Desert

\section{Introduction}

Humans have, either directly (through introduction) or indirectly, caused the range expansion of a great number of species, which can result in detrimental impacts on native organisms and their ecosystems (Cox 1999, Simberloff 2005). Usually invasive species are of exotic origin, but sometimes a species may be native to a region, and, through the actions of humans, increase drastically in population size and range. Often it is unclear whether the increase is due to in situ population increase, invasion of the region by individuals from peripheral populations, or some combination of the two means. Genetic methods have great potential to help unravel the history of an invasive population by enabling measurements of gene flow among populations, documenting secondary colonizations (e.g., Fonseca et al. 2006), estimating long and short-term trends in population size (Wang 2005), and even unraveling the potential role of selection in 
invasion success (Lee 2002). Such results, in combination with traditional methods of population analysis, can help to identify the factors responsible for population change.

In the Mojave desert region of the Southwestern U.S., populations of common ravens have virtually exploded over the past half century, with perhaps as much as a 15fold increase just in the past three decades (Boarman 1993; Boarman and Berry 1995, Liebezeit and George 2004). This increased abundance of ravens is a direct response to increased human impacts in the region, including subsidizations of water and food from agriculture, development, landfills and road kills, and creation of additional nesting sites (e.g., telephone poles, electric transmission towers, planted trees and bridges). Raven reproductive success is enhanced by proximity to anthropogenic subsidies (Kristan and Boarman in press), juvenile survival is higher in nests near human habitations (Webb et al. 2004), and reproductive output is higher in birds with garbage contained in their castings (Kristan et al. 2004). The increase in raven numbers also correlates with increased predation on desert tortoises (Gopherus agassizii, a federally threatened species under the U. S. Endangered Species Act), and undoubtedly impacts other native species upon which the common raven preys.

Clearly, humans have impacted the survival and reproductive success of common ravens in the Mojave Desert, but it is not known to what extent raven populations in these regions have been increased through immigration from surrounding areas. In spite of the apparent success in this region, preliminary demographic analyses suggest that Mojave raven populations are not sustainable without immigration from some other area (Boarman and Kristan 2006). Ravens have a prodigious capability of flight, with some birds having daily commutes to landfills of up to $65 \mathrm{~km}$. Juvenile dispersal distances in 
the desert region were found to average $8.6 \mathrm{~km}$ for males and $8.8 \mathrm{~km}$ for females (Boarman, unpublished), but both natal and breeding dispersal events up to about $320 \mathrm{~km}$ have been observed in other regions (Boarman and Heinrich 1999). Recent broad-scale phylogeographic analyses (Omland et al. 2000, 2006) suggest that ravens may have a higher level of philopatry than would be expected based on their powers of flight and reported studies of individual movements. Our study provides genetic assessments on more local scales to help shed light on the source of population increase and long-term effectiveness of management efforts (USFWS 2007) to reverse those trends. In addition, the documented population growth in the Mojave affords comparison of census estimates of growth with trends we estimate from genetic data.

In this paper, we assess levels and patterns of variation in both mitochondrial DNA control region sequences and nuclear microsatellite loci. We then estimate a range of genetic and demographic parameters for the West and East Mojave Desert raven populations, including estimates of genetic variation within and among populations, rates of gene flow, and tests of population size change. We interpret our findings to determine whether the population increase in the Mojave Desert is detectable by genetic analyses, whether we can differentiate how much of the growth may be due to in situ population growth versus immigration or expansion from neighboring regions, and from which direction (i.e., coastal northern, southern or Central Valley of California, or Great Basin of Nevada) this migration may occur.

\section{Materials and methods:}

Sampling 
We obtained blood or tissue samples from adult ravens from collecting localities nested within five regions of California or Nevada (Fig. 1): (1) West Mojave Desert (WMoj), individual birds were trapped, banded and bled from the U. S. Army National Training Center, Fort Irwin, San Bernardino County, California $(n=34)$ and Edwards Air Force Base, Kern County, CA ( $=32)$; (2) East Mojave Desert (EMoj), where birds were salvaged from two localities in Clark County, southern Nevada: Boulder City Landfill (N 35.96471 by W 114.80894$)(\mathrm{n}=29)$ and Laughlin Landfill (N 35.18230 by $\mathrm{W}$ 114.63502) ( $\mathrm{n}=15)$; (3) northern Nevada (Great Basin; NoNV), where we obtained salvaged tissues or feather samples from birds collected as part of raven control programs from five counties (Washoe, Elko, Humboldt, Lander and Mineral; $n=25$ ); (4) coastal southern California (SoCA), salvaged or museum tissue samples from San Diego $(n=6)$ and Los Angeles/Riverside Counties $(n=6) ;(5)$ and museum tissue samples from coastal northern California (all from the San Francisco Bay area in Marin, San Mateo, Alameda, Solano and Contra Costa counties, $\mathrm{n}=16$; NoCA). An additional three samples were obtained from three sites in the Central Valley of California, utilizing raven control programs and museum tissue collections, but the limited sample size precluded extensive analyses of this sample.

\section{DNA methods}

DNA was isolated from whole blood, tissue or fresh feather samples using Qiagen DNeasy kits following the manufacturer's protocol. A 314 bp portion of the mitochondrial DNA control region was amplified and sequenced for 105 Nevada and California common ravens using primers Cor-Lg12 and H417 and protocols described in 
Tarr and Fleischer (1999) and Omland et al. (2000). In addition, 24 sequences from California populations obtained for earlier studies (Tarr and Fleischer 1999; Omland et al. 2000) were incorporated into the analyses (see Fig. 2 legend for Genbank accession numbers).

A suite of eight microsatellite loci was also amplified from 163 samples from these localities and analyzed for size variation on an ABI 3100 sequencer (Table 1). Primers for seven of these loci (CK1B5D, CK1B6G, CK2A5A, CK4A3G, CK4B6D, CK5A4B, and CK5A5F) were originally developed for the Marianas crow (Corvus kubaryi; Tarr and Fleischer 1998) and one (MJG1) was developed for the Mexican jay (Aphelocoma ultramarina; Li et al. 1997). We used laboratory conditions as described in Tarr and Fleischer (1998) and Omland et al. (2000) for the microsatellite amplifications, with the annealing temperatures provided in Table 1. All of the Marianas crow primers are dinucleotides (CA). The MJG1 locus normally contains a tetrameric repeat (GAAA) in the Mexican and other jays (Li et al. 1997; Lillandt et al. 2002; Fleischer et al., unpublished), but was found to contain a pentameric (GAAAA) repeat in the common raven. In addition, a number of MJG1 alleles were scored at an unexpected size (i.e., they were not sized five base pairs apart from the next smaller or larger allele). All individuals with unexpected allele sizes were re-amplified and rerun at least one additional time to confirm the allele size. In addition, we cloned and sequenced a subset of these, and some alleles of expected pentanucleotide motif, to confirm that the sizes called by the genotyping program matched the prediction from the actual sequence. This also allowed us to determine whether alleles of the same size have identical sequences (i.e. no evidence of size homoplasy), which was generally the case. In cases where 
alleles did not match the size expected from a pentanucleotide repeat the difference was due to an increase or reduction in the size of one or more repeats (e.g., from GAAAA to GAAA or GAAAAA, etc.).

\section{Analyses of Genetic Structure}

We constructed a minimum spanning haplotype network from 129 mtDNA control region sequences (58 from Nevada and 71 from California) using the program TCS 1.21

(Clement et al. 2000). We analyzed levels and patterns of variation in mtDNA control region sequences using Arlequin v. 3.0 (Excoffier et al. 2005). We computed nucleotide diversity $(\pi)$ and haplotype diversity $(\mathrm{H})$ within regions, and Tajima's D, Fu's F and mismatch distributions to assess population size trends locally and across all regions. We conducted AMOVA's in Arlequin to assess genetic differentiation among sampling sites nested within the two regions (i.e., California and Nevada) and between the two regions, as well as $\mathrm{F}_{\mathrm{ST}}$ directly among sampling areas and regions. We also computed these values and tests for only the California clade ( $\mathrm{n}=32$ for Nevada and 69 for California), and for the Holarctic clade ( $\mathrm{n}=25$ for Nevada and two for California). More extensive phylogeographic analyses suggest that Holarctic clade haplotypes might have entered the southwestern U.S. more recently, and that the California clade haplotypes were in residence for a much longer period of time (Omland et al. 2000). As noted above, for some analyses we combined the sampling sites into only two regions (i.e., Nevada and California) when there were no significant differences between sampling sites within regions and we wanted increased power to estimate parameters. 
We estimated long-term effective population sizes from estimates of $\theta$ from mtDNA control region sequence data using Migrate 2.1 (Beerli and Felsenstein 1999, 2001). Because of concerns raised by Abdo et al. (2004), Slatkin (2005), and other authors about overestimation of $\mathrm{Nm}$ in Migrate, especially with small values of $\theta$ and low sample sizes, we did not use the program to estimate asymmetries in migration among populations. We ran analyses using a DNA sequence mutation model and Bayesian inference. For the Bayesian analysis we ran five independent replicate analyses of three long chains each; each long chain was run for 100,000 trees, with the first 10,000 trees discarded as unreliable "burn-in". We retained default parameters and starting estimates based on $\mathrm{F}_{\mathrm{ST}}$ estimates.

For microsatellites, we used Genepop v. 1.2 (Raymond and Rouset 1995) and Arlequin v. 3.0 (Excoffier et al. 2005) to estimate expected and observed heterozygosity and to test for deviations from expectations of Hardy-Weinberg equilibrium. Genepop was also used to test for linkage disequilibrium among loci. Differentiation of allele frequencies across populations was tested with a Markov chain Fisher Exact Test in Genepop. The inbreeding coefficients $\mathrm{F}_{\mathrm{ST}}$ and $\mathrm{R}_{\mathrm{ST}}$ were estimated from allele frequencies under infinite alleles and stepwise mutation assumptions, respectively, in Arlequin, and tested using permutations for significant difference from zero. However, we present only $\mathrm{F}_{\mathrm{ST}}$ because of concerns of accuracy of $\mathrm{R}_{\mathrm{ST}}$ estimates given our sample sizes (Gaggliotti et al. 1999). We assessed the deviation in observed heterozygosity from that expected under mutation-drift equilibrium using the coalescent program Bottleneck 1.2.02 (Piry et al. 2000); this program assesses the likelihood of a recent $\left(t=2 N_{e}\right)$ population bottleneck. We used a two-phased mutation model (TPM) set at 90\% stepwise and 10\% infinite 
alleles mutation, and tested the deviation using a non-parametric Wilcoxon test. The Bayesian analysis program Structure 2.1 (Pritchard et al. 2000) was used to determine the number of distinct populations to which individuals could be assigned based on their multilocus genotype. We ran analyses without prior information on population identity, with both independent and correlated allele frequencies, and used four replicates of 10,000 runs for "burn-in" and sampling from 100,000 replicates to assess likelihoods for $\mathrm{k}$ from 1 to 5 populations. We also analysed the microsatellite dataset with the program Migrate 2.1 (Beerli and Felsenstein 1999, 2001) as we did for mtDNA; we used the stepwise mutation model and 100,000 trees per chain (with a burn-in of 10,000). We obtained Bayesian estimates of $\theta$ within each sampling area.

\section{Results}

\section{MtDNA analyses}

We obtained up to $314 \mathrm{bp}$ (range 286 to $314 \mathrm{bp}$ ) of mtDNA control region sequence for each of 129 ravens from California and Nevada (Figs. 1 and 2). In total, there were 38 distinct haplotypes and 51 variable sites. The sequences, as expected from previous work (Tarr and Fleischer 1999; Omland et al. 2000), separated into two divergent clades: 11 haplotypes (27 individuals) in the Holarctic clade and 27 haplotypes (102 individuals) in the California clade. TCS1.21 (Clement et al. 2000) produced a haplotype network (Fig. 2) with only one ambiguous connection in the Holarctic clade, but a sizeable number of ambiguous connections in the California clade. All but two of the 71 raven sequences from California fell into the California clade (i.e., two birds from Fort Irwin were in the Holarctic clade), whereas a little more than half of the 58 Nevada raven sequences fell 
into the California clade $(50.0 \%$ of the northern Nevada ravens and $61.8 \%$ of the southern Nevada ravens; Fig. 2). Control region sequences recovered included 23 haplotypes that had been found in prior studies and their Genbank accession numbers are listed in the legend to Fig. 2; Genbank accession numbers for 15 novel sequences are underlined.

We found that mtDNA sequence variability differs among sampling sites, states and clades (Table 2). Sampling sites within Nevada have more haplotypes and higher values of haplotype diversity $(H)$, nucleotide diversity $(\pi)$ and $\theta$. These differences are caused, in part, by the equal occurrence of haplotypes of both clades in Nevada, while the California populations contain haplotypes primarily (Fort Irwin) or only from a single clade. However, when considering the California clade alone, there are 11 haplotypes among 12 individuals from northern Nevada populations $(\mathrm{H}=0.985$ and $\pi=$ 0.012 \pm 0.007$), 11$ haplotypes among 22 individuals in the East Mojave Desert sampling area $(H=0.924$ and $\pi=0.009 \pm 0.006)$, and only 14 haplotypes among 66 individuals from California populations $(\mathrm{H}=0.865$ and $\pi=0.009 \pm 0.005)$, suggesting that the differences in diversity between the states are not due only to the equal proportions of haplotypes from the two divergent clades in Nevada.

To assess the possibility of population change or selection we also calculated Tajima's D, Fu's Fs, and goodness of fit statistics to mismatch distributions from the sequences within each sampling site and state (Table 2). Only the WMoj sampling area had a significant difference from zero for Tajima's D $(-1.52, \mathrm{p}<0.05)$, suggesting either recent population expansion or purifying selection in this population. In addition, the mismatch distribution for WMoj is not significantly different from predictions of a sudden expansion model $(\mathrm{p}=0.08)$. The mismatch distribution for NoCA strongly 
indicated a bottleneck, with highly significant deviations from the expansion model. D was significantly negative for all California sampling areas combined (Table 2), possibly because the West Mojave Desert sample overwhelmed the smaller samples from coastal California. Non-significant, positive D's for the two Nevada sampling areas suggest no change in population size or selection in this region. However, when the analysis is conducted on California clade haplotypes alone, both Fs and D are negative in both NoNV and EMoj, significantly so for Fs in both cases $(F s=-5.0, p=0.008$ and $-3.9, p=$ 0.018, respectively). As before, for the three California sampling areas, Tajima's D and Fu's Fs are significantly negative only in WMoj, but not for either SoCA or NoCA (Table 1). When conducted on the Holarctic clade haplotypes alone for the NoNV and the EMoj sampling areas, neither Tajima's D nor Fu's Fs were significantly different from zero, and mismatch distributions were significantly different from an expanding population model ( $\mathrm{p}=0.03$ and 0.05 , respectively). Thus analysis of the California clade provides evidence of population expansion in both regions, but the Holarctic clade shows evidence of population stasis in Nevada.

The minimum spanning network produced by TCS (Fig. 2) shows no evidence of reciprocal monophyly among sampling sites or regions, nor fixed differences in haplotype frequencies. Nonetheless, the populations showed a relatively high level of haplotypic frequency differentiation as assessed by $\mathrm{F}_{\mathrm{ST}}$ and AMOVA. When all samples were grouped into either a California or a Nevada sample $\mathrm{F}_{\mathrm{ST}}$ was $0.214(\mathrm{p}<0.0000)$, indicating a relatively low rate of gene flow between the states $(\mathrm{Nm}=1.84$ migrants per generation). When the five sampling areas were grouped hierarchically by state (NV = NoNV and EMoj; CA = WMoj, NoCA and SoCA) and analysed by AMOVA we 
recovered a relatively high and significant $\Phi_{\mathrm{ST}}$ value among sampling areas $\left(\Phi_{\mathrm{ST}}=0.246\right.$, $\mathrm{p}<0.0001$ ), and this was further divided into a $\Phi_{\mathrm{SC}}$ among sample regions within states of $0.073(\mathrm{p}=0.014)$, and higher component of the variation $\Phi_{\mathrm{CT}}$ between states of 0.186 $(\mathrm{p}<0.0001)$. Pair-wise $\mathrm{F}_{\mathrm{ST}}$ values calculated among sampling regions are shown in Table 3, along with estimates of $\mathrm{Nm}$ from Arlequin. When the two Mojave sampling regions are combined into a group, $\Phi_{\mathrm{SC}}$ increases to 0.172 , suggesting that the two Mojave Desert sites are not a natural group. In addition, when assessing the structure of the haplotype network (Fig. 2) or tree (not shown), haplotypes from Nevada and California often occur in separate "subclades" and are more often connected to members of their own region than might be expected by chance. The difference between the regions is not due only to the higher presence of Holarctic clade haplotypes in Nevada: $\Phi_{\mathrm{ST}}$ across the sampling areas for just the California clade is $0.229(\mathrm{p}<0.0001)$; and for just the Holarctic clade is $0.107(\mathrm{p}=0.26) . \mathrm{F}_{\mathrm{ST}}$ for Holarctic clade haplotypes between the northern Nevada and East Mojave Desert sampling areas is only $0.008(\mathrm{p}=0.29)$, indicating high mtDNA similarity of the two populations and reflecting a rate of gene flow, $\mathrm{Nm}$, of 65.2 individuals per generation.

\section{Microsatellite analyses}

We analyzed variation in eight microsatellite loci (Table 1) for 163 individuals (mean recovery of 158.4 individuals per locus). The microsatellite loci varied in level of polymorphism, averaging 11.5 alleles per locus (range from 4 to 43 alleles) and $68.8 \%$ observed heterozygosity. Mean heterozgosity for microsatellites was highest in the Mojave Desert sampling areas and lowest in $\operatorname{NoCA}(\mathrm{EMoj}=0.720 ; \mathrm{WMoj}=0.683$; 
NoNV $=0.626 ;$ SoCA $=0.622 ;$ NoCA $=0.557)$, but did not differ significantly among sampling areas. As for mtDNA, we combined the five sampling sites in northern Nevada into one sampling region $(n=23)$; there were no significant differences among the sites in allele frequencies. We conducted preliminary analyses on this and seven other locality samples (NoNV, Laughlin, Boulder City, Fort Irwin, Edwards AFB, NoCA, SoCA and the Central Valley; Fig. 1). Because of their proximity, and a lack of significant differences in allele frequencies between them, we combined the Laughlin and Boulder City as the EMoj sampling region $(n=44)$, and the Fort Irwin $(n=32)$ and Edwards AFB $(\mathrm{n}=34)$ samples as the WMoj region $(\mathrm{n}=66)$. We divided our other California samples into NoCA $(n=16)$ and SoCA $(n=11)$, and Central Valley $(n=3)$ samples. There was no significant difference in allele frequencies between San Diego and Los Angeles area samples $(\mathrm{p}=0.35)$.

Observed heterozygosity at all eight loci in each of the eight locality samples did not significantly deviate from expectations of Hardy-Weinberg equilibrium with the exception of locus CK2A5A in NoNV ( $p=0.007)$ and CK1B6G in NoCA $(p=0.026)$. These loci were in Hardy-Weinberg equilibrium in the other populations and this deviation may reflect the broad geographic sampling across NoNV, or just be a chance deviation. There was no evidence for linkage disequilibrium in the microsatellite dataset; only a single test out of 224 disequilibrium tests (eight loci in eight populations) was significant (CK4B6D and CK5A5F in NoNV, $\mathrm{p}=0.034)$. In addition, it is very unlikely that birds from a sampling site were more related to each other than random: we analysed microsatellite genotypes using the program Kinship 1.3.1 (Goodnight and Queller 1999) within each larger locality sample in Nevada and California, and found 
only a small fraction of pairwise comparisons of individuals had values of $\mathrm{R}$ indicative of first-order relationship (1.7\% for Boulder City; 1.9\% for Laughlin; 3.0\% for Fort Irwin, and $4.1 \%$ for Edwards AFB). For samples in northern Nevada and southern and northern California, samples were not taken at a single time or site, so incidental sampling of close relatives is very unlikely.

Only the NoCA sample showed a significant excess heterozygosity in comparison to that expected under mutation-drift equilibrium and a two-phase mutation model (Wilcoxon test, $\mathrm{p}=0.0098$; Piry et al. 1999). This significant result suggests that the northern California population may have undergone a recent bottleneck. This test does have low power with small sample sizes of individuals ( $\mathrm{n}=16$ for this sample) and loci (only eight), but the mtDNA data also suggested a bottleneck in NoCA, as does the lower mean heterozygosity in the NoCA sample.

All loci except CK5A4B showed significant variation in allele frequencies across five sampling regions $(\mathrm{p}<0.0014)$. An AMOVA, grouping the populations into Nevada and California, revealed low divergence among groups within states $\left(\mathrm{F}_{\mathrm{SC}}=0.019\right)$ or among states $\left(\mathrm{F}_{\mathrm{CT}}=0.005\right)$. Pair-wise $\mathrm{F}_{\mathrm{ST}}$ values were considerably lower than those calculated for mtDNA, a pattern that may be caused by the very high mutation rates in these markers (Hedrick 1999, Epperson 2005; see discussion). $\mathrm{F}_{\mathrm{ST}}$ ranged from 0.003 to 0.042 across the eight loci (Table 4), but most values were significantly different from zero based on permutation tests in Arlequin v3.0. $\mathrm{F}_{\mathrm{ST}}$ values indicate that genetic divergence is highest between the NoNV and other sample areas (mean $\mathrm{F}_{\mathrm{ST}}=0.032 \pm$ 0.005), and was very low between SoCA and the West Mojave Desert $\left(\mathrm{F}_{\mathrm{ST}}=0.003\right)$. In spite of the significant $\mathrm{F}_{\mathrm{ST}}$ values, Bayesian analysis of genetic structure using Structure 
2.1 (Pritchard et al. 2000) with no prior population identification did not support differentiation into more than one population (i.e., $\mathrm{k}=1$ ), nor show any clustering of individuals geographically (data not shown).

\section{Estimating gene flow and $N_{e}$}

Gene flow estimates were calculated in Arlequin using Slatkin's (1995) method (Table

3). These values are very high between NoNV and EMoj and SoCA and the WMoj, but are small between the EMoj and WMoj samples, and between NoCA and WMoj. Gene flow estimates from microsatellite data were on average higher and showed a mostly similar pattern to those from mtDNA (Table 4). Gene flow was very high between SoCA and the WMoj, but not as high between NoNV and the EMoj, and perhaps greater than expected for other comparisons in relation to the rates obtained from the mtDNA analyses. Whitlock and McCauley (1998), among others, have pointed out the problems of untested assumptions and sampling error in estimating gene flow rates from $\mathrm{F}_{\mathrm{ST}}$ and its analogues, so we provide these as relative estimates only.

Values of $\theta$ estimated from mtDNA control region sequences using Migrate 2.1.3 were generally higher in the Nevada populations $(\mathrm{NoNV}=0.0234 ; \mathrm{Emoj}=0.0417)$ relative to the California ones $(\mathrm{Wmoj}=0.0243$; $\mathrm{SoCA}=0.0038 ; \mathrm{NoCA}=0.0026)$. Estimates of $\theta$ from microsatellites were also calculated using a Bayesian approach in Migrate 2.1.3, and were also higher inland $(\mathrm{NoNV}=0.6107 ; \mathrm{EMoj}=1.1051 ; \mathrm{WMoj}=$ $2.0103)$ than along coastal California $(\mathrm{SoCA}=0.6555 ; \mathrm{NoCA}=0.3572)$. If we assume a mean mutation rate of $10^{-7}$ for mtDNA CR (e.g., Omland et al. 2006), the long-term effective population size $\left(\mathrm{N}_{\mathrm{e}}\right)$ estimates based on $\theta=2 \mathrm{~N}_{\mathrm{e}} \mu$ are relatively small in coastal 
California $(\mathrm{NoCA}=13,000 ;$ SoCA $=19,000)$ and considerably larger in the desert regions $(\mathrm{WMoj}=121,500 ; \mathrm{EMoj}=208,500 ; \mathrm{NoNV}=117,000)$. For microsatellites, if we assume a mean mutation rate of $10^{-4}$ (range of $10^{-2}$ to $10^{-4}$; Epperson 2005) the longterm effective population size $\left(\mathrm{N}_{\mathrm{e}}\right)$ estimates based on $\theta=4 \mathrm{~N}_{\mathrm{e}} \mu$ mostly match in pattern, but are considerably smaller in magnitude $(\mathrm{NoCA}=1,786 ; \mathrm{SoCA}=3,277 ; \mathrm{WMoj}=$ 10,065; EMoj $=5,525 ;$ and NoNV $=3,054$ individuals). These order of magnitude differences in effective population size estimates between markers could be a result of an incorrect mutation rate for either marker, i.e., if the microsatellite mutation rate is $10^{-5}$ or the mtDNA mutation rate is $10^{-6}$, these estimates would converge. Alternatively, the differences may be caused by a very different mutational timeframe for the two marker classes (mtDNA measuring a considerably longer period in the past than microsatellites), or there just could be considerable measurement error in one or both datasets.

\section{Discussion}

Genetic Structure

Our mitochondrial DNA control region analyses revealed a surprising degree of genetic structure in common raven populations in the southwestern U. S., and less gene flow among the Great Basin deserts of Nevada, the Mojave Desert and coastal regions of California than might be expected based on the flight capabilities of ravens and availability of habitat. The high AMOVA $\mathrm{F}_{\mathrm{ST}}$ value among sampling areas $(=0.246)$ for mtDNA control region sequences indicates a fairly low level of overall maternal gene flow, as does the high percentage of private haplotypes (41.9\% overall, with the highest values in the two Nevada sample regions). The pattern of pairwise $\mathrm{F}_{\mathrm{ST}}$ values (Table 3) 
and shared haplotypes among sampling areas (Fig. 2) indicates high gene flow between the West Mojave Desert and coastal southern California sampling areas $\left(\mathrm{F}_{\mathrm{ST}}=0.027, \mathrm{~ns}\right)$, and between the East Mojave Desert and northern Nevada $\left(\mathrm{F}_{\mathrm{ST}}=0.008, \mathrm{~ns}\right)$, but not between the East and West Mojave Desert sampling areas $\left(\mathrm{F}_{\mathrm{ST}}=0.141, \mathrm{p}<0.001\right)$. The northern California sampling area is as divergent from all the other regions as the Nevada sample (Table 3).

The microsatellite data, on the other hand, did not reveal as many private alleles within sampling regions $(9.8 \%)$, and produced $\mathrm{F}_{\mathrm{ST}}$ values that were considerably smaller in value (mean of $0.022, \mathrm{p}<0.0001$ ) than those calculated from mtDNA sequences. They showed low and non-significant differentiation between the West Mojave Desert and southern California sampling areas $\left(\mathrm{F}_{\mathrm{ST}}=0.003\right)$, but significant divergence between the West Mojave Desert and the other three sampling areas. In general, the analyses of gene flow based on microsatellites and mtDNA provide a roughly similar pattern of gene flow among sampling areas (with the exception of relatively low gene flow between northern Nevada and East Mojave Desert ravens), but very different magnitudes. Similar discrepancies in the values of $\mathrm{F}_{\mathrm{ST}}$ between mtDNA and nuclear microsatellite datasets have been found for other avian and vertebrate taxa (e.g., Gibbs et al. 2000, Haavie et al. 2000, Chappell et al. 2004, Tiedemann et al. 2004). Milot et al. (2000) estimated an $\mathrm{F}_{\mathrm{ST}}$ of 0.53 for mtDNA control region sequences across the range of the yellow warbler (Dendroica petechia), while the $\mathrm{F}_{\mathrm{ST}}$ calculated from microsatellites for the same population samples was only 0.014 (Gibbs et al. 2000). And Tiedemann et al. (2004) found an $\mathrm{F}_{\mathrm{ST}}$ of about 0.502 for mtDNA control region and only 0.053 for microsatellites among colonies of eider ducks (Somateria mollissima). 
This discrepancy in divergence levels based on F-statistics between mtDNA and microsatellites could indicate that nuclear alleles are moving more between regions than the maternally inherited mtDNA haplotypes (high female philopatry). Alternatively, because coalescence or sorting of nuclear alleles occurs at a slower rate than for mtDNA haplotypes, the lower differentiation may reflect an earlier stage of divergence. Hedrick (1999) notes that $\mathrm{F}_{\mathrm{ST}}$ values calculated using highly mutable and variable markers should underestimate $\mathrm{F}_{\mathrm{ST}}$, and provides a method (equation $2 \mathrm{a}$ ) to calculate the maximum $\mathrm{F}_{\mathrm{ST}}$ value expected for rapidly evolving markers such as microsatellites. For our raven sample this is 0.288 . Thus, the highest $\mathrm{F}_{\mathrm{ST}}$ we obtain of 0.042 actually represents 14.6 percent of the maximum theoretical value. Epperson (2005) has recently quantified the divergence underestimates for genetic markers with very high mutation rates (such as microsatellites) relative to those with slower rates (such as mtDNA). Some, if not all, of our microsatellites may fall into this hypermutable category (especially MJG1, Table 1). Alternatively, if the hypothesis of reduced mtDNA gene flow is correct, it means that female ravens are dispersing much less than males, and this is unexpected from existing demographic data for both ravens and other songbirds, in which females usually disperse more often and further (Boarman, unpublished; Clarke et al. 1997). But if males are dispersing at high rates, we should still see less mtDNA divergence because they carry their mtDNA with them when they move.

\section{Population Size Changes and Gene Flow}

Calculations of Tajima's D and Fu's Fs from our mtDNA sequence data provide evidence of raven population growth in the Mojave Desert but population stasis in the Great Basin 
in Nevada and southern California. When this was dissected by clade, there was evidence of expansion for the California clade in both California and Nevada, but evidence of stasis for Holarctic clade samples in Nevada. This suggests that birds from the California clade may have been expanding into the Great Basin, where Holarctic clade members existed, although results from our structure analyses do not completely confirm this. Our bottleneck analysis of microsatellite variation reveals evidence of a bottleneck only for the NoCA sample. The evidence for Mojave Desert population growth matches expectations based on historical data on population trends for this region (Boarman 1993, Boarman and Berry 1995, Liebezeit and George 2004). Ravens were rare in most parts of the Mojave Desert up until the past fifty years. Once humans began to provide excess food and nesting sites with their garbage, road-killed animals and power lines, raven reproductive success and population size increased dramatically (Knight et al. 1993, Boarman 1993, Webb et al. 2004). The great increase in numbers of ravens in the Mojave Desert may be due to in situ increases from such subsidies, or to invasion of ravens from adjacent regions (or some combination of the two). Anthropogenic resources facilitate survival of ravens in the Mojave Desert, but modeling enigmatically suggests that the populations are not self-sustaining, and can only be maintained or increased via immigration (Boarman and Kristan 2006). This result may be a partial artifact of the high level of mortality caused by illegal poisoning of mostly juveniles by dairy farmers in the area where the above study took place (WIB, personal observation).

Our assessment of genetic structure was able to shed only limited light on whether the West Mojave Desert common raven population increase was due to in situ population 
growth, immigration, or both factors combined. Our results did indicate that if an invasion was the cause of the massive population increase in the West Mojave Desert rather than in situ growth, it likely did not occur from Nevada and the Great Basin or Northern California. The WMoj ravens are genetically very similar to those in SoCA and there is a high level of gene flow between them, thus SoCA would seem to be the likely invasion source. However we cannot rule out the inverse: that the WMoj ravens were the source of movement into southern California. In support of this idea is the finding that genetic variation in mtDNA (Table 2) and microsatellites are much lower in SoCA than WMoj (although so is sample size). But this would also be expected if SoCA is not the only source population for WMoj, and we cannot rule out an expansion from the Central Valley into the WMoj. Indeed, the three individuals samples from the Central Valley all share haplotypes with the WMoj sample. The higher variation in the WMoj could reflect the mixed nature of the population arising from movement from SoCA and the Central Valley. Another finding of our genetic structure analyses is that there appears to be relatively limited gene flow between the EMoj and WMoj sampling areas.

There is a good deal of what is considered high quality raven habitat between the western Mojave Desert, the Central Valley and coastal California (WIB, personal observation). Hence there should be high connectivity and opportunity for movement among these regions (i.e, from the Los Angeles basin or southern Central Valley into the Mojave Desert). However, much of the East Mojave Desert region, between the Great Basin and the West Mojave Desert, is extremely arid, has much lower use by humans, and consists of suboptimal habitat for common ravens, and would likely represent an obstacle to dispersal for ravens. Human habitations (isolated homesteads, small 
communities, roadside rests) that provide important resources for ravens, may serve as stepping-stones for dispersal and wanderings of ravens through the desert (Boarman and Kristan in prep.). Furthermore, ravens nesting farther from anthropogenic resources tend to have more seeds and insect in their diets and have lower reproductive success (Kristan et al. 2004). While there has been considerable anthropogenic development on the edge of the eastern Mojave Desert in southern Nevada (i.e., Las Vegas/Clark County), there is very little human development in the region between there and the western Mojave. As development occurs in this region, in particular along interstate highways leading to Las Vegas, it may open up a corridor or conduit for raven nesting and dispersal that would lead to increased movement of ravens between the eastern and western Mojave.

\section{Management and policy implications}

Our results have implications for management of raven populations in the Mojave Desert and other parts of its expansive range. The USFWS is currently developing plans to reduce raven predation on tortoises through lethal and non-lethal means. It is important for them to know if limiting reproductive and feeding opportunities in the Mojave Desert will reduce raven population size and density, or whether immigration will nullify such management actions? Clearly, our genetic data show the possibility of large-scale movements with reproduction (i.e., gene flow). This evidence of large-scale, region wide movements supports the need for regional-scale management efforts rather than just local ones. Current gaps in anthropogenic habitat are probably the only factor maintaining the genetic structure we identified. Developments and other actions that subsidize essential resources for ravens provide more hospitable habitat thereby facilitating the incursion of 
ravens farther into the desert. The presence of more ravens increases their predation pressure on desert tortoises and other species of concern (Kristan and Boarman 2003).

We were able to use genetic analysis of contemporary populations to gain some understanding of the dynamics of population growth and colonization in an invasive species. Genetic analysis has been used to assess the origins, demography and consequences of biological invasions for plant (e.g., Meekins et al. 2000, Novak 2004), invertebrate (e.g., Ingram and Gordon 2003), lower vertebrate (e.g., Estoup et al. 2004, Kolbe et al. 2004) and bird species (e.g., Clegg et al. 2000; Hawley et al. 2006). But common ravens in the West Mojave Desert of California also represent an unusual case in that they are a native species in the region, but also apparently an invasive one, that acts as a pest, and as a potentially limiting factor on threatened populations of desert tortoises. We used genetic markers to detect signals of population growth within this region, and to exclude some potential regions as the likely source populations (Great Basin, Northern California), but not others (i.e., Southern California, Central Valley). These results illustrate the utility of population genetic analyses to help uncover or elucidate past demographic events in populations affected by human activities.

\section{Acknowledgments}

We thank M. Pavelka, K. Brock, M. Vermeys, P. Coater, J. Peter, B. Miller, D. Rowley, D. Capelli, M. Holmgren (UC Santa Barbara), K. Burns (San Diego State University), K. Garrett (LACO), J. Dumbacher (CAS), A. Engilis (UC Davis), and C. Cicero (MVZ) for assistance with collecting, or providing, tissue samples. Mark Hagan and Edwards Air Force Base provided funding and logistical support for a portion of the work. Thanks 
also to M. Haynie and F. Hailer for advice on data analysis. E. G. G was sponsored by a predoctoral fellowship of the Ministerio de Educación y Ciencia from Spain.

\section{References}

Abdo Z, Crandall KA, Joyce P. (2004) Evaluating the performance of likelihood methods for detecting population structure and migration. Molecular Ecology 13, 837-51.

Beerli P, Felsenstein J (1999) Maximum-likelihood estimation of migration rates and effective population numbers in two populations using a coalescent approach. Genetics 152, 763-773.

Beerli P, Felsenstein J (2001) Maximum likelihood estimation of migration rates and effective population numbers in two populations using a coalescent approach. Proceedings of the National Academy of Sciences, USA 98, 4563-4568.

Beerli P (2006) Comparison of Bayesian and maximum-likelihood inference of population genetic parameters. Bioinformatics 22, 341-345.

Boarman WI (1993) When a native predator becomes a pest: a case study. Pages. 191-206 in S. K. Majumdar, E. W. Miller, D. E. Miller, E. K. Brown, J. R. Pratt, and R. F. Schmalz, editors. Conservation and resource management. Pennsylvania Academy of Science, Philadelphia, Pennsylvania, USA.

Boarman WI, Berry KH (1995) Common Ravens in the Southwestern United States, 1968-92. Pp.73-75 In: Laroe, E.T., ed. Our living resources: A report to the nation on the distribution, abundance, and health of U.S. plants, animals, and ecosystems. National Biological Service, Washington, D.C.

Boarman WI, Heinrich B (1999) Common Raven (Corvus corax), In The Birds of North America, No. 476 (Poole A, Gill F, eds.). The Birds of North America, Inc., Philadelphia, PA.

Boarman WI, Kristan WB (2006) Trends in Common Raven Populations in the Mojave and Sonoran Deserts: 1968-2004. Report to U.S. Fish and Wildlife Service, Ventura, CA. Contract No: 814405M055.

Chappell DE, Van Den Bussche RA, Krizan J, Patterson B (2004) Contrasting levels of genetic differentiation among populations of wolverines (Gulo gulo) from northern Canada revealed by nuclear and mitochondrial loci. Conservation Genetics 5, 759-767.

Clarke, AL, Saether B-E, Roskaft E. 1997. Sex biases in avian dispersal: a reappraisal. Oikos 79, 429-438.

Clegg SM, Degnan SM, Kikkawa J, Moritz C, Estoup A, Owens IPF (2002) Genetic 
consequences of sequential founder events by an island-colonizing bird. Proceedings of the National Academy of Sciences, USA 99, 8127-8132.

Clement M, Posada D, Crandall KA (2000) TCS: a computer program to estimate gene genealogies. Molecular Ecology 9, 1657-1659.

Cox GW (1999) Alien species in North America and Hawaii: impacts on natural ecosystems. Washington, DC, Island Press.

Epperson BK (2005) Mutation at high rates reduces spatial structure within populations. Molecular Ecology 14, 703-710.

Estoup A, Beaumont M, Sennedot F, Moritz C, Cornuet J-M (2004) Genetic analysis of complex demographic scenarious: spatially expanding populations of the cane toad Bufo marinus. Evolution 58, 2021-2036.

Excoffier L, Laval G, Schneider S (2005) Arlequin ver. 3.0: An integrated software package for population genetics data analysis. Evolutionary Bioinformatics Online 1, 4750 .

Fonseca DM, Smith JL, Wilkerson RC, Fleischer RC (2006) Pathways of expansion and multiple introductions illustrated by large genetic differentiation among worldwide populations of the southern house mosquito. American Journal of Tropical Medicine and Hygiene 74, 284-289.

Gaggiotti OE, Lange O, Rassmann K, Gliddon C (1999) A comparison of two indirect methods for estimating average levels of gene flow using microsatellite data. Molecular Ecology 8, 1513-1520.

Gibbs HL, Dawson RJG, Hobson KA (2000) Limited differentiation in microsatellite DNA variation among northern populations of the yellow warbler: evidence for malebiased gene flow? Molecular Ecology 9, 2137-2147.

Goodnight KF, Queller DC (1999) Computer software for performing likelihood tests of pedigree relationship using genetic markers. Molecular Ecology 8, 1231-1234.

Haavie J, Sætre G, Moum T (2000) Discrepancies in population differentiation at microsatellites, mitochondrial DNA and plumage colour in the pied flycatcher inferring evolutionary processes. Molecular Ecology 9, 1137-1148.

Harpending H C (1994) Signature of ancient population growth in a low-resolution mitochondrial DNA mismatch distribution. Human Biology 66, 591-600.

Hawley DM, Hanley D, Dhondt AA, Lovette IJ (2006) Molecular evidence for a founder effect in invasive house finch (Carpodacus mexicanus) populations experiencing an emergent disease epidemic. Molecular Ecology 15, 263-275. 
Hedrick P (1999) Perspective: Highly variable loci and their interpretation in evolution and conservation. Evolution 53, 313-318.

Ingram KK, Gordon DM (2003) Genetic analysis of dispersal dynamics in an invading population of Argentine ants. Ecology 84, 2832-2842.

Knight RL, Kawashima JY (1993) Response of raven and red-tailed hawk populations to linear right-of-ways. Journal of Wildlife Management 57, 266-271.

Kolbe JJ, Glor RE, Rodríguez Schettino L, Chamizo Lara A, Larson A, Losos JB (2004) Genetic variation increases during biological invasion by a Cuban lizard. Nature 431, 177-181.

Kristan WB III, Boarman WI (2003) Spatial distribution of risk of desert tortoise (Gopherus agassizii) predation by common ravens (Corvus corax). Ecology 84, 24322443.

Kristan WB III, Boarman WI, Crayon J (2004) Diet composition of common ravens across the urban-wildland interface of the west Mojave Desert. Wildlife Society Bulletin 32, 244-253.

Li S-H, Huang Y-J, Brown JL (1997) Isolation of tetranucleotide repeat microsatellites from the Mexican jay. Molecular Ecology 6, 499-501.

Lee CE (2002) Evolutionary genetic of invasive species. Trends in Ecology and Evolution 17, 386-391.

Liebezeit JR, George TL (2004) A summary of predation by corvids on threatened and endangered species in California and management recommendations to reduce corvid predation. 145 pp. Humboldt State University, Arcata, CA.

Lillandt BG, Bensch S, Hansson B, Wennerberg L \& Von Schantz T (2002) Brief report - Isolation and cross-species amplification of microsatellite loci in the Siberian jay (Perisoreus infaustus). Hereditas 137, 157-160.

Meekins JF, Ballard Jr. HE, McCarthy BC (2000) Genetic variation and molecular biogeography of a North American invasive plant species (Alliaria petiolata, Brassicaceae). International Journal of Plant Sciences 162, 161-169.

Milot E., Gibbs HL, Hobson KA (2000) Phylogeography and genetic structure of northern populations of the yellow warbler (Dendroica petechia). Molecular Ecology 9 , 667-682.

Novak SJ (2004) Genetic analysis of downy brome (Bromus tectorum) and medusahead (Taeniatherum caput-medusae): management implications. Weed Technology 18, 1417- 
1421.

Omland KE, Tarr CL, Boarman WI, Marzluff JM, Fleischer RC (2000) Cryptic genetic variation and paraphyly in ravens. Proceedings of the Royal Society of London. Series B, Biological Sciences, 267, 2475-2482.

Omland KE, Baker JM, Peters JL (2006) Genetic signatures of intermediate divergence: population history of Old and New World Holarctic ravens (Corvus corax). Molecular Ecology, 15, 795-808.

Piry S, Luikart G, Cornuet JM (1999) Bottleneck: a computer program for detecting recent reductions in the effective population size using allele frequency data. Journal of Heredity 90, 502-503.

Pritchard JK, Stephens M, Donnelly P (2000) Inference of population structure using multilocus genotype data. Genetics, 155, 945-959

Raymond M., Rousset F. 1995. GENEPOP (version 1.2): population genetics software for exact tests and ecumenicism. Journal of Heredity, 86, 248-249

Simberloff D (2005) Non-native species do threaten the natural environment! Journal of Agricultural and Environmental Ethics 18, 595-607.

Slatkin M (1995) A measure of population subdivision based on microsatellite allele frequencies. Genetics 139, 457-462.

Slatkin M (2005) Seeing ghosts: the effect of unsampled populations on migration rates estimated for sampled populations. Molecular Ecology, 14, 67-73.

Tarr, CL, Fleischer RC (1998) Primers for polymorphic GT microsatellites isolated from Mariana crow, Corvus kubaryi. Molecular Ecology, 7, 253-255.

Tarr CL, Fleischer RC (1999) Population boundaries and genetic diversity in the endangered Mariana crow (Corvus kubaryi). Molecular Ecology, 8, 941-950.

Tiedemann R, Paulus KB, Scheer M, Von Kistowski KG, Skirnisson K, Bloch D, Dam M (2004) Mitochondrial DNA and microsatellite variation in the eider duck (Somateria mollissima) indicate stepwise postglacial colonization of Europe and limited current longdistance dispersal. Molecular Ecology 13, 1481-1494.

U.S. Fish and Wildlife Service (2007) Draft environmental assessment to implement a desert tortoise recovery plan task: reduce common raven predation on the desert tortoise. Ventura Field Office, Ventura, CA.

Wang J (2005) Estimation of effective population sizes from data on genetic markers. Philosophical Transactions of the Royal Society of London B, 360, 1395-1409. 
Webb WC, Boarman WI, Rotenberry JT (2004) Common raven juvenile survivorship in a human augmented landscape. Condor 106, 517-528.

Whitlock MC, McCauley DE (1999) Indirect measures of gene flow and migration: FST_1/(4Nm+1). Heredity 82, 117-125. 
Table 1 Microsatellite loci used in the study (Tarr and Fleischer 1998; Li et al. 1997), number of alleles, observed heterozygosity (calculated in Genepop v. 1.2; Raymond and Rouset 1995) and annealing temperature during PCR. Mean and standard error on bottom line.

\begin{tabular}{lccc} 
& No. & & Anneal \\
Locus & alleles & $\mathrm{H}_{0}$ & Temp ${ }^{\circ} \mathrm{C}$ \\
\hline CK1B5D & 8 & 0.827 & 60 \\
CK1B6G & 4 & 0.578 & 55 \\
CK2A5A & 8 & 0.631 & 55 \\
CK4A3G & 4 & 0.654 & 55 \\
CK4B6D & 6 & 0.490 & 55 \\
CK5A4B & 11 & 0.765 & 62 \\
CK5A5F & 7 & 0.662 & 55 \\
MJG1 & 44 & 0.900 & 55 \\
\hline Mean & $11.5 \pm 4.7$ & $0.688 \pm 0.048$ &
\end{tabular}


Table 2 Statistics of variation in mtDNA sequences from the five sampling areas and divided by state (see Fig. 1).

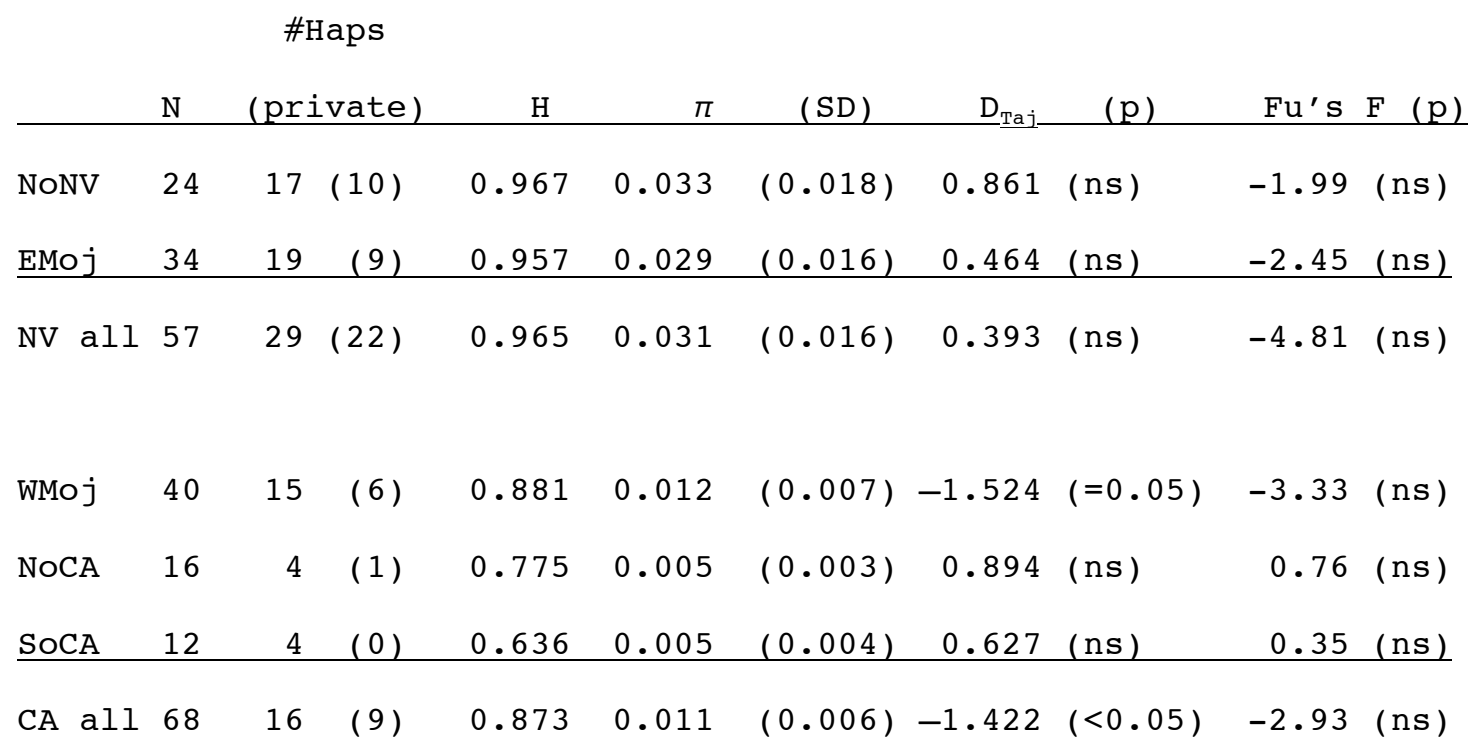


Table 3 Population pair-wise $\mathrm{F}_{\mathrm{ST}}$ values below the diagonal, and migration (Nm; number of migrants per generation) values above the diagonal, for the five sampling areas in Nevada and California calculated from mtDNA control region haplotype frequencies. $\mathrm{F}_{\mathrm{ST}}$ values significantly different from zero are indicated by astericks $(* * \mathrm{p}<0.01$, and $* * * \mathrm{p}<$ $0.001)$.

$\begin{array}{lllllr} & \text { NONV } & \text { EMOj } & \text { WMOj } & \text { NOCA } & \text { SoCA } \\ \text { NONV } & \underline{0.000} & 58.40 & 1.41 & 0.94 & 1.40 \\ \text { EMOj } & 0.008 & \underline{0.000} & 3.03 & 1.29 & 3.05 \\ \text { WMOj } & 0.261 * * * & 0.141 * * * & \underline{0.000} & 1.61 & 17.96 \\ \text { NOCA } & 0.348 * * * & 0.279 * * * & 0.238 * * * & \underline{0.000} & 0.35 \\ \text { SOCA } & 0.263 * * * & 0.141 * * & 0.027 & 0.585 & \underline{0.000}\end{array}$


Table 4 Population pair-wise $\mathrm{F}_{\mathrm{ST}}$ values below the diagonal, and migration (Nm; number of migrants per generation) values above the diagonal, for the five sampling areas in Nevada and California, calculated from microsatellite allele frequencies. $\mathrm{F}_{\mathrm{ST}}$ values significantly different from zero are indicated by astericks $(* \mathrm{p}<0.05, * * \mathrm{p}<0.01$, and $* * * \mathrm{p}$ $<0.001)$.

$\begin{array}{lllccc} & \text { NONV } & \text { EMOj } & \text { WMOj } & \text { NOCA } & \text { SOCA } \\ \text { NONV } & \underline{0.000} & 7.8 & 6.2 & 15.8 & 5.7 \\ \text { EMOj } & 0.032 * * * & \underline{0.000} & 10.5 & 18.0 & 18.6 \\ \text { WMOj } & 0.039 * * * & 0.023 * * * & \underline{0.000} & 8.1 & 72.6 \\ \text { NOCA } & 0.016 * & 0.014 * * & 0.029 * * * & \underline{0.000} & 11.3 \\ \text { SOCA } & 0.042 * * & 0.013 & 0.003 & 0.021 * & \underline{0.000}\end{array}$




\section{Figure Legends}

Fig. 1 Map of the southwestern United States showing the Mojave Desert and the approximate localities of common raven sampling sites in California and Nevada. The five major sampling regions are in boldface and underlined. Sample sizes are total numbers of birds analysed, but differ between mtDNA and microsatellite analyses.

Fig. 2 Common raven mitochondrial DNA control region haplotype network constructed with the program TCS1.21 (Clement et al. 2000). Sequences divide into two major clades (Holarctic and California), expected based on previous work (Tarr and Fleischer 1999, Omland et al. 2000, 2005). The area of each colored pie diagram reflects the number of individuals of each haplotype (also given as " $n$ ") and colored segments represent the proportion of the number of haplotypes that occur from each sampling region. Note only two Holarctic clade haplotypes and individuals were found in California sampling areas. Haplotype Genbank accession numbers are listed by haplotype number (underlined are novel haplotypes discovered in this study): 1 AF115304, 2 AY710371, 3 AY005894, 4 EU031791, 5 EU031792, 6 AY710416, 7 AY005872, 8 AY005919, 9 EU031793, 10 EU031794, 11 EU031795, 12 AY005883, 13 AY005882, 14 pending, 15 AY710374, 16 AY710428, 17 EU031796, 18 EU031797, 19 AF115300, 20 AY005880, 21 AY005871, 22 EU031798, 23 EU031799, 24 AF115298, 25 AF115301, 26 AF115302, 27 pending, 28 AY005876, 29 AY005897, 30 AF115304, 31 EU031801, 32 EU031802, 33 EU031803, 34 AY005893, 35 AY710412, 36 AY005886, 37 EU031804, 38 EU031805. 


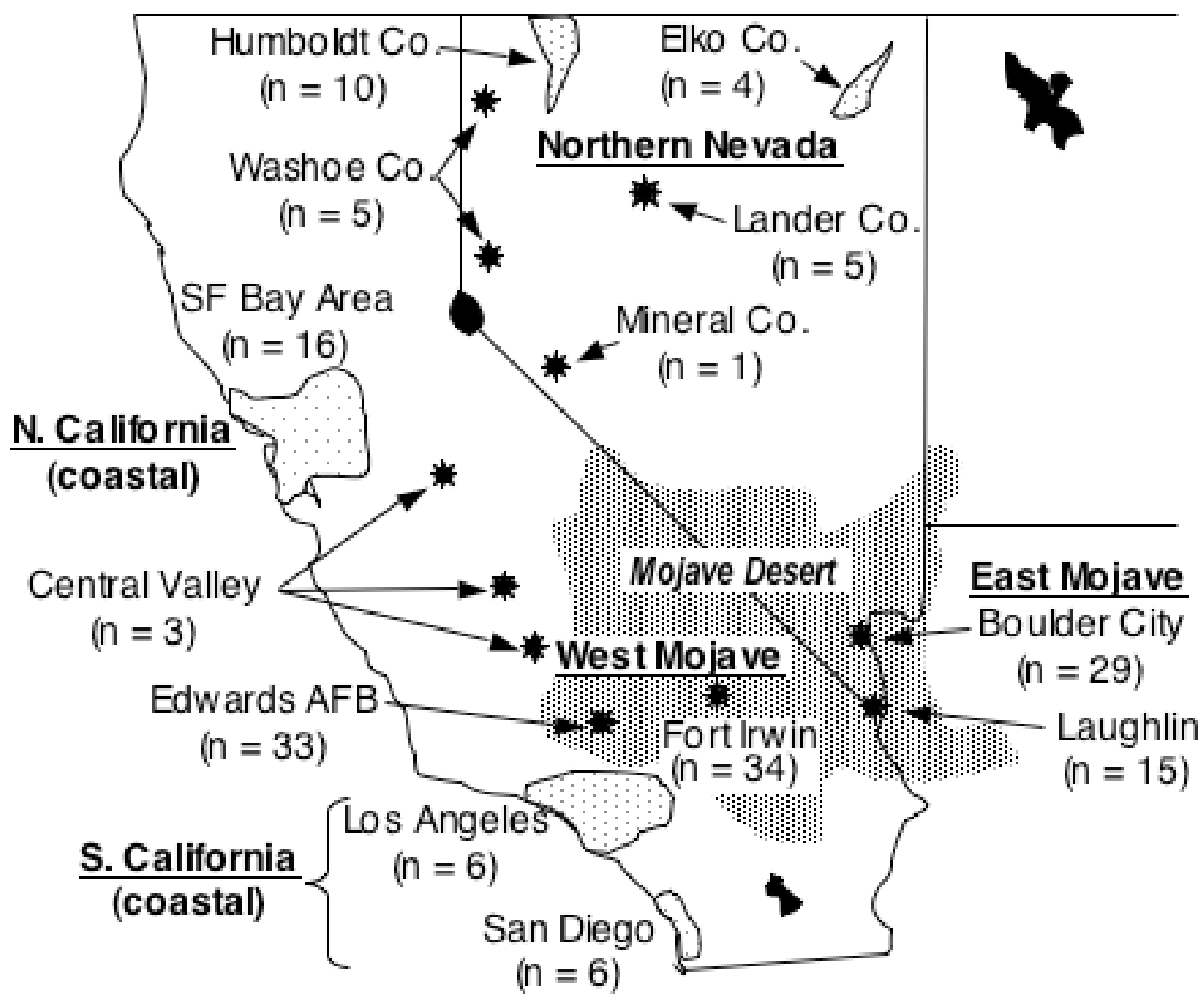


Northern Nevada $(n=24)$

East Mojave Desert (Nevada; $n=34$ )

West Mojave Desert (California; $n=40$ )

Northern California (coast; $n=16$ )

Southern California (coast; $n=12$ )

Central Valley (California; $n=3$ )

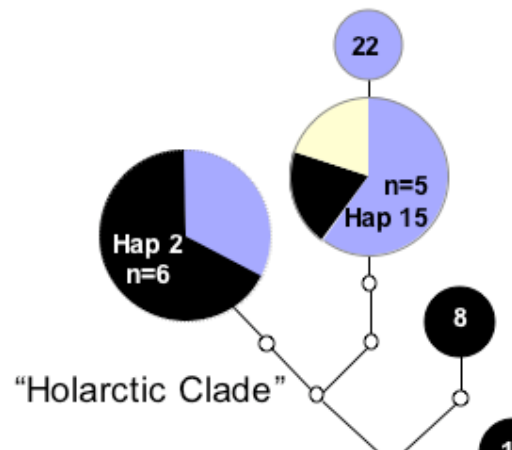

29
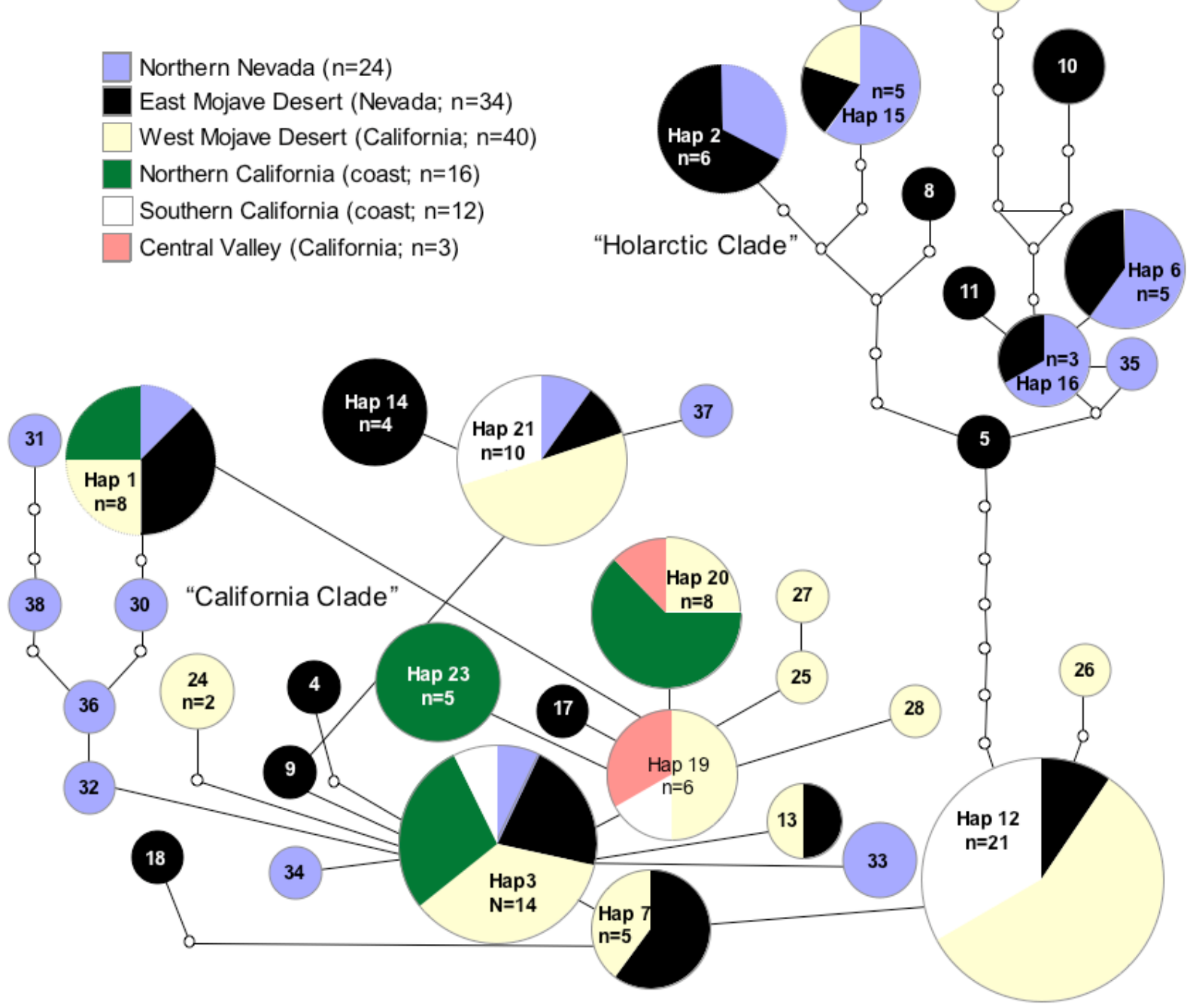